\title{
A Comparison of Modern Hyperbolic Methods for Semiconductor Device Simulation: NTK Central Scheme Vs. CLAWPACK
}

\author{
CARL L. GARDNER*, ANNE GELB ${ }^{\dagger}$ and JUSTIN HERNANDEZ \\ Department of Mathematics, Arizona State University, Tempe AZ 85287-1804, USA \\ (Received 1 May 2001; Revised 1 April 2002)
}

\begin{abstract}
Two modern hyperbolic methods-a second-order Godunov method in the software package CLAWPACK and the second-order Nessyahu-Tadmor-Kurganov (NTK) central scheme-are compared for simulating an electron shock wave in the classical hydrodynamic model for semiconductor devices.

The NTK central scheme, which does not employ Riemann problem solutions, is described in detail. Special attention is paid in both methods to handling the source terms in the hydrodynamic model. CLAWPACK incorporates the source terms by a splitting method, while our version of the NTK scheme is unsplit.
\end{abstract}

Keywords: Numerical methods for hyperbolic PDEs; CLAWPACK; Hyperbolic central schemes; NTK central scheme

\section{INTRODUCTION}

The classical hydrodynamic model has become a standard industrial simulation tool which incorporates important "hot electron" phenomena in submicron semiconductor devices. The hydrodynamic model consists of non-linear hyperbolic conservation laws for particle number, momentum, and energy (with a heat conduction term), coupled with Poisson's equation for the electrostatic potential. The non-linear hyperbolic modes support shock waves-"velocity overshoot" in the parlance of semiconductor device physicists.

To accurately compute solutions including sharp resolution of shock waves, we implemented the hydrodynamic model equations in LeVeque's software package CLAWPACK ${ }^{\ddagger}$ (Conservation LAWs PACKage) and in our general gas dynamical code which uses the NessyahuTadmor-Kurganov (NTK) central scheme [1]. CLAWPACK consists of routines for solving time-dependent nonlinear hyperbolic conservation laws based on higher order
Godunov methods and (approximate) Riemann problem solutions, while the NTK scheme solves conservation laws using a modified Lax-Friedrichs central difference method without appealing to Riemann problem solutions.

We will compare hydrodynamic model steady-state simulations (including shock waves) using CLAWPACK and the NTK central scheme of the $n^{+}-n-n^{+}$diode, which models the channel of a field effect transistor. Steady state is achieved by simulating in time to equilibrium.

A splitting method is used to incorporate the source terms in the hydrodynamic model for CLAWPACK, while our version of the NTK scheme is unsplit. In both numerical codes, we use an implicit method for the source terms.

\section{THE CLASSICAL HYDRODYNAMIC MODEL}

The hydrodynamic model treats the propagation of electrons in a semiconductor device as the flow of a charged compressible fluid. The model plays an important

\footnotetext{
*Corresponding author. Tel.: + 1-480-965-0226. Fax: + 1-480-965-0461E-mail: gardner@asu.edu. Research supported in part by the National Science Foundation under grant DMS-9706792.

${ }^{\dagger}$ E-mail: ag@math.la.asu.edu. Research supported in part by the Sloan Foundation and in part by the National Science Foundation under grant DMS-0107428.

"http://www.amath.washington.edu/ claw/. R.J. LeVeque's CLAWPACK website.
} 
role in predicting the behavior of electron flow in semiconductor devices since it exhibits hot carrier effects missing in the standard drift-diffusion model. The hydrodynamic description should be valid for devices with active regions $\gtrsim 0.05$ microns.

The hydrodynamic model is equivalent to the equations of electro-gas dynamics:

$$
\begin{gathered}
\frac{\partial}{\partial t}(m n)+\frac{\partial}{\partial x_{i}}\left(m n u_{i}\right)=0 \\
\frac{\partial}{\partial t}\left(m n u_{j}\right)+\frac{\partial}{\partial x_{i}}\left(m n u_{i} u_{j}\right)+\frac{\partial P}{\partial x_{j}}=-n \frac{\partial V}{\partial x_{j}}-\frac{m n u_{j}}{\tau_{p}} \\
\frac{\partial W}{\partial t}+\frac{\partial}{\partial x_{i}}\left(u_{i}(W+P)+q_{i}\right) \\
=-n u_{i} \frac{\partial V}{\partial x_{i}}-\frac{\left(W-\frac{3}{2} n T_{0}\right)}{\tau_{w}} \\
\nabla \cdot(\epsilon \nabla V)=e^{2}(N-n)
\end{gathered}
$$

where $m$ is the effective electron mass, $n$ is the electron density, $u_{i}$ is the velocity, $m n u_{i}$ is the momentum density, $P=n T$ is the pressure (Boltzmann's constant $k_{B}$ is set equal to 1$), T$ is the temperature, $V=-e \phi$ is the potential energy, $\phi$ is the electrostatic potential, $e>0$ is the electronic charge,

$$
W=\frac{3}{2} n T+\frac{1}{2} m n u^{2}
$$

is the energy density, $q_{i}$ is the heat flux, and $T_{0}$ is the temperature of the semiconductor lattice. Indices $i, j$ equal 1, 2, 3, and repeated indices are summed over. Equation (1) expresses conservation of electron number (or mass), Eq. (2) expresses conservation of momentum, Eq. (3) expresses conservation of energy, and Eq. (4) is Poisson's equation. The last terms in Eqs. (2) and (3) represent electron scattering, which is modeled by the standard relaxation time approximation, with momentum and energy relaxation times $\tau_{p}$ and $\tau_{w}$.

The non-linear conservations laws Eqs. (1)-(3) are simply the Euler equations of gas dynamics for a charged gas with polytropic gas constant $\gamma=5 / 3$ in a electric field, with the addition of heat conduction and relaxation terms.

In this investigation we take $\tau_{p}$ and $\tau_{w}$ to be constant; we also set the heat flux $q_{i}=0$ since we are interested here in numerical methods which sharply resolve shock waves. Note that the hydrodynamic model equations have source terms on the right-hand sides of Eqs. (2) and (3) from the relaxation time terms and from the coupling to the electric field $\mathbf{E}=-\nabla \phi$.

The electron gas has a soundspeed and the electron flow may be either subsonic or supersonic. In general a shock wave develops at the transition from supersonic flow to subsonic flow.

\section{THE $n^{+}-n-n^{+}$DIODE}

The hydrodynamic model has been extensively used to study the $n^{+}-n-n^{+}$diode which models the channel of a field effect transistor. The diode begins with a heavily doped $n^{+}$source region, followed by a lightly doped $n$ channel region, and ends with an $n^{+}$drain region.

Hydrodynamic model simulations of a steady-state electron shock wave [2] in a one micron $\mathrm{Si}$ semiconductor device at $77 \mathrm{~K}$ were validated in Ref. [3] by a Monte Carlo simulation of the Boltzmann equation using the DAMOCLES program [4]. A shock profile develops in the channel as the supersonic flow on entering the channel breaks to a subsonic flow, just like gas dynamical flow in a Laval nozzle. The electron shock wave is analogous to the gas dynamical shock wave in the Laval nozzle, where the $n^{+}-n-n^{+}$doping of the diode corresponds to the converging/diverging geometry of the Laval nozzle.

The steady-state upwind shock simulations presented in Ref. [2] were reproduced in Ref. [5] using a timedependent "essentially non-oscillatory" (ENO) upwind scheme, a higher order Godunov method.

The shock computations imply that the electron shock waves are an integral part of the hydrodynamic model. The shock waves allow higher electron velocities to develop in the channel, and provide a richer space charge structure in the device.

For the transonic computations presented below, we take a GaAs diode at $T_{0}=300 \mathrm{~K}$ with a 0.25 micron source, a 0.25 micron channel, and a 0.25 micron drain. In the $n^{+}$region, the doping density $N=5 \times 10^{17} \mathrm{~cm}^{-3}$, while in the $n$ region $N=2 \times 10^{15} \mathrm{~cm}^{-3}$. In GaAs, the effective electron mass $m=0.063 m_{e}$ at $300 \mathrm{~K}$, where $m_{e}$ is the electron mass, and the dielectric constant $\epsilon=12.9$. We set $\tau_{p}=\tau_{w}=0.2$ picoseconds. Since the flow is subsonic at inflow and outflow, we impose the boundary conditions $n=N$ at the left and right, $T=T_{0}$ at the left, and $\phi=0$ at the left and $\phi=1$ volt at the right.

\section{CLAWPACK}

Our main interest here lies with the non-linear hyperbolic modes of the hydrodynamic model, since they support shock waves. Implementing a higher order Godunov method via CLAWPACK yields sharp resolution of these discontinuities.

CLAWPACK is second-order accurate in regions of smooth flow and first-order accurate near discontinuities for the homogeneous conservation laws of gas dynamics. The best results were obtained using the Roe approximate Riemann solver and the van Leer flux limiter.

The hydrodynamic model has inhomogeneous source terms and we use a splitting method to take them into account. The one-dimensional hydrodynamic model conservation laws take the form

$$
q_{t}+f(q)_{x}=\psi(q)
$$


where the conserved quantities $q=(m n, m n u, W)$, the flux

$$
f(q)=\left(m n u, m n u^{2}+P, u(W+P)\right.
$$

and the source terms

$$
\psi(q)=-\left(0, e n E+\frac{m n u}{\tau_{p}}, e n E+\frac{W-\frac{3}{2} n T_{0}}{\tau_{W}}\right) .
$$

LeVeque [6] suggests using a standard splitting or fractional step method in which one alternates between solving the homogeneous equation

$$
q_{t}+f(q)_{x}=0
$$

and the ordinary differential equation (ODE)

$$
q_{t}=\psi(q)
$$

We use the second-order accurate implicit trapezoidal rule method for solving the ODE [Eq. (10)] to insure accuracy and stability. Non-linear source terms are handled by Newton iteration.

Since the hydrodynamic model equations are coupled with Poisson's equation for the electrostatic potential, a elliptic solve must also be performed. To discretize Poisson's equation (4) we use a second-order accurate central difference for the approximation of the second derivative $V_{x x}$. The potential is then updated by means of a standard direct tridiagonal solver.

Solving the hydrodynamic model Eqs. (1)-(4) over one timestep $\Delta t$ involves the following steps:

1. Solve the source term ODE [Eq. (10)] over a timestep $\Delta t / 2$.

2. Using step 1 as initial data, solve the gas dynamical homogeneous conservation laws [Eq. (9)] over $\Delta t$.

3. Solve Poisson's equation (4), using the results from step 2 as initial data.
4. Solve the source term ODE [Eq. (10)] over a timestep $\Delta t / 2$, using the previous results in steps 2 and 3 as initial data.

The Strang splitting yields second-order accurate results; in practice steps 1 and 4 are combined except in the first and last timesteps.

Using CLAWPACK, we performed simulations of the $n^{+}-n-n^{+}$diode with $300 \Delta x$. The CLAWPACK results are compared in the Figures with the results of the NTK central scheme with $300 \Delta x$.

Figures 1-5 present the simulation of the electron shock wave. The shock wave develops at $x \approx 0.2$ microns in Fig. 1 and with CLAWPACK is spread out over approximately one or two $\Delta x$ (see Fig. 2).

Figure 3 displays the doping profile $N$ and steady-state solution for electron density $n$. Because of the high density contrast of the background doping, the shock wave in density is downplayed. Note the slight cooling in Fig. 4 as the electrons overcome a small potential barrier at the first junction, and the dramatic increase in temperature as the electrons heat up in the channel before entering the drain. Figure 5 depicts the effects on the electric field of the electron shock wave and of the space charge $n-N$ at the junctions.

\section{NTK CENTRAL SCHEME}

High resolution non-oscillatory central schemes have recently become popular in solving non-linear hyperbolic conservations laws. We first consider the one-dimensional scalar conservation law

$$
u_{t}+f(u)_{x}=0
$$

The computation is performed over cells of width $\Delta x=x_{j+1 / 2}-x_{j-1 / 2}$ given by the piecewise constant

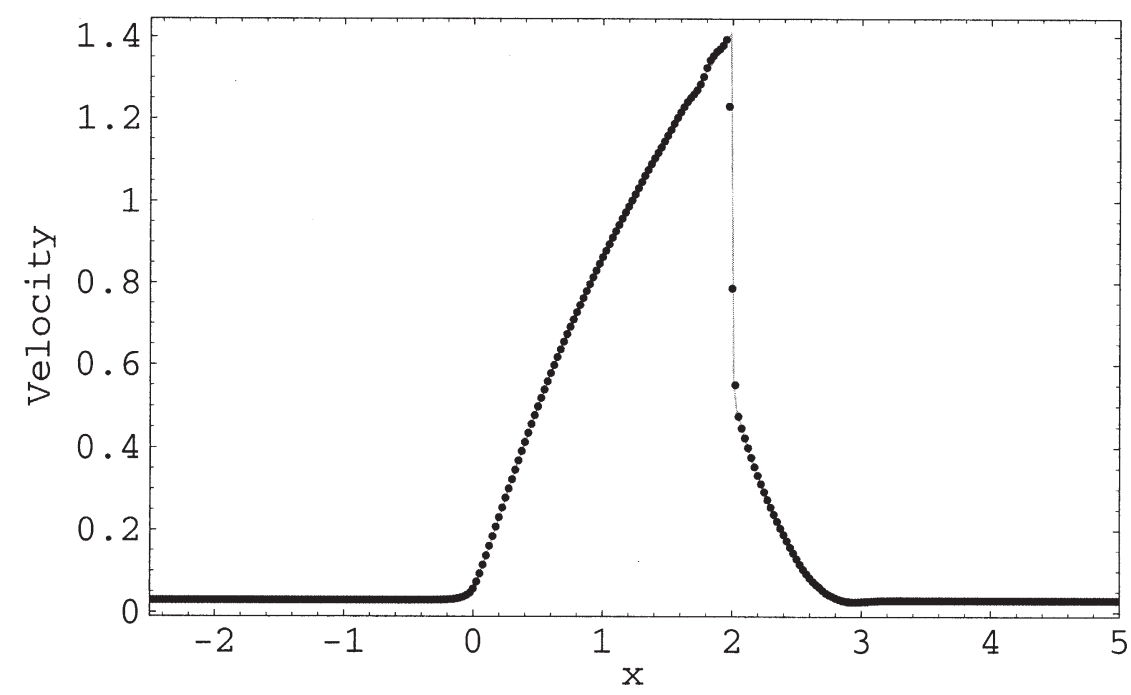

FIGURE 1 Electron velocity in $10^{7} \mathrm{~cm} / \mathrm{s}$ using CLAWPACK (solid) vs. the NTK central scheme (dotted solution values). $x$ is in 0.1 microns for all figures. 


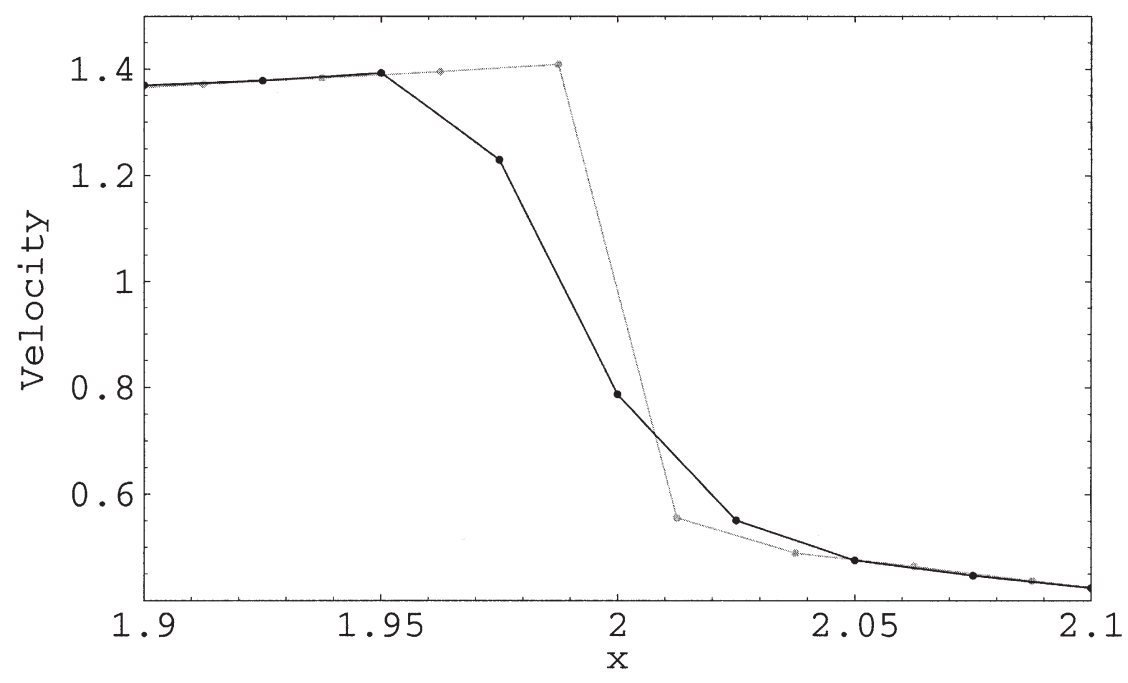

FIGURE 2 Closeup of the electron shock wave in velocity.

approximate solution

$$
\bar{u}(x, t)=u_{j}(t), \quad x_{j-\frac{1}{2}} \leq x \leq x_{j+\frac{1}{2}}
$$

where gridpoints are labeled by $j=0,1, \ldots, N$. The original Nessyahu-Tadmor (NT) scheme [7] was derived from the prototypical staggered version of the LaxFriedrichs $(\mathrm{LxF})$ scheme,

$$
\begin{aligned}
u_{j+\frac{1}{2}}(t+\Delta t)= & \frac{1}{2}\left(u_{j}(t)+u_{j+1}(t)\right)-\lambda\left[f\left(u_{j+1}(t)\right)\right. \\
& \left.-f\left(u_{j}(t)\right)\right]
\end{aligned}
$$

where $\lambda=\Delta t / \Delta x$. As noted in Ref. [7], central schemes integrate over the entire Riemann fan, taking into account both left and right traveling waves. Hence there is no need to employ a Riemann solver. By exploiting this freedom, we show how high resolution central schemes can be effectively adapted to numerically solve conservation laws with source terms. Specifically, since no Riemann solvers need to be employed for the homogeneous hyperbolic part of the system, the source terms can be handled implicitly in an unsplit way, thereby reducing the overall computational time. This technique will be demonstrated in the application of a high resolution central scheme to the hydrodynamic model for semiconductor devices. If the source terms are non-linear, then Newton iteration can be performed, which now involves repeating the gas dynamical step as well. This repetition will be computationally expensive, so a semi-implicit method for the source terms may be substituted.

The idea behind the NT scheme is to reduce the amount of inherent dissipation in the LxF scheme [Eq. (13)]. This is accomplished by replacing the piecewise constantsolutions in Eq. (12) with piecewise linear approximations

$$
L_{j}(x, t)=u_{j}(t)+\left(x-x_{j}\right) \frac{u_{j}^{\prime}}{\Delta x}, \quad x_{j-\frac{1}{2}} \leq x \leq x_{j+\frac{1}{2}}
$$

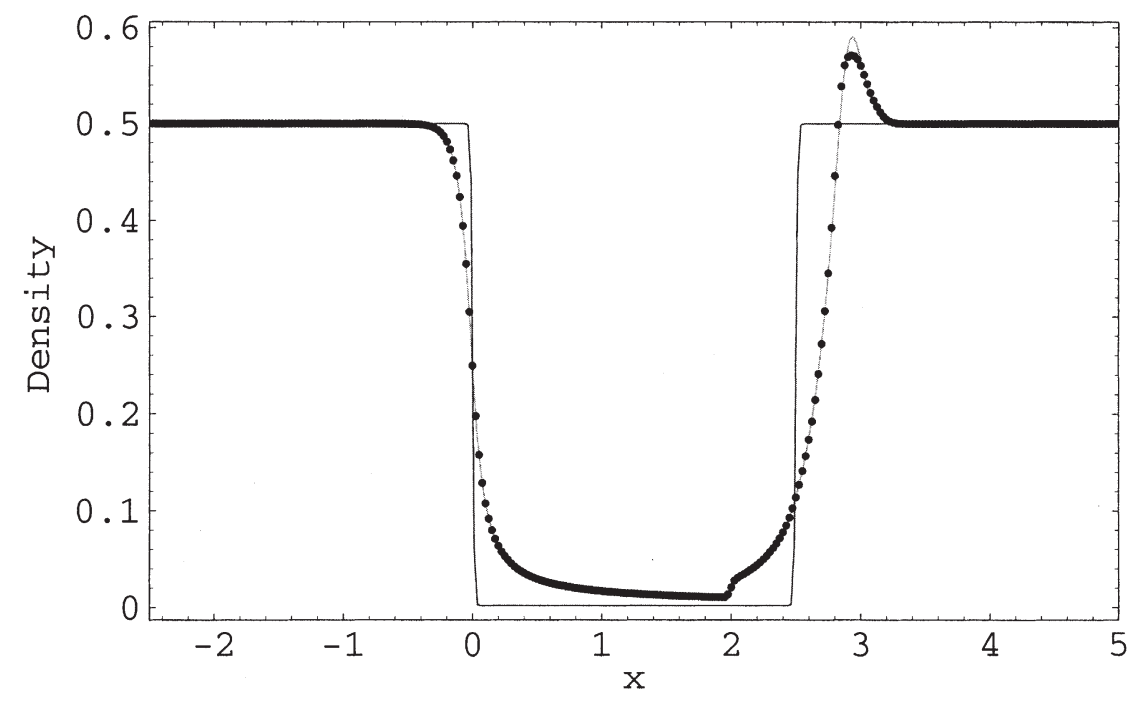

FIGURE 3 Electron density using CLAWPACK (solid) vs. the NTK central scheme (dotted) and doping profile in $10^{18} \mathrm{~cm}^{-3}$. 


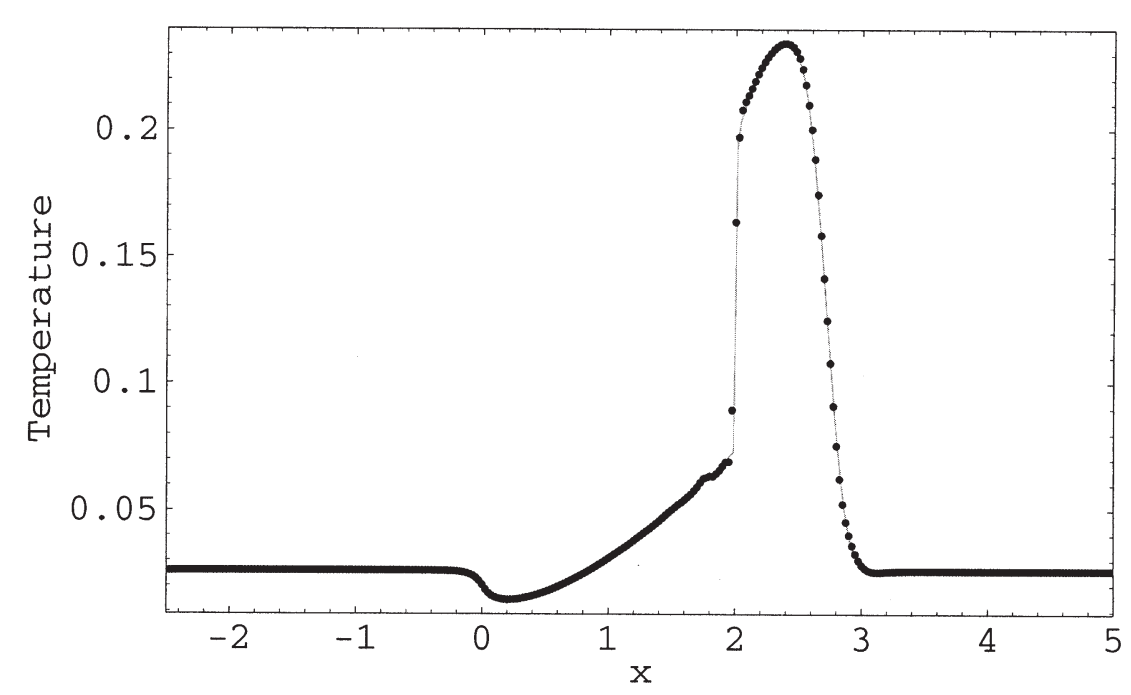

FIGURE 4 Electron temperature in electron volts using CLAWPACK (solid) vs. the NTK central scheme (dotted).

where the numerical derivative at $x_{j}$ is $u_{j}^{\prime} / \Delta x$. A secondorder scheme is derived by evolving this linear interpolant in time to produce

$$
\begin{gathered}
u_{j+\frac{1}{2}}(t+\Delta t)=\frac{1}{2}\left(u_{j}(t)+u_{j+1}(t)\right)+\frac{1}{8}\left(u_{j}^{\prime}-u_{j+1}^{\prime}\right) \\
-\lambda\left[f\left(u_{j+1}\left(t+\frac{\Delta t}{2}\right)\right)-f\left(u_{j}\left(t+\frac{\Delta t}{2}\right)\right)\right] .
\end{gathered}
$$

The CFL condition is satisfied by

$$
\lambda \max _{x}\left|\frac{\partial f}{\partial u}\right|<\frac{1}{2} .
$$

Analogously for systems we have

$$
\lambda \max _{x} \rho(A(u(x, t)))<\frac{1}{2}
$$

where $\rho(\cdot)$ denotes the spectral radius and $A(u)$ is the Jacobian $\partial f / \partial u$ of the flux.

By Taylor expansion, the midpoint values can be approximated by

$$
u_{j}\left(t+\frac{\Delta t}{2}\right)=u_{j}-\frac{1}{2} \lambda f_{j}^{\prime}
$$

where the numerical flux derivative at $x_{j}$ is $f_{j}^{\prime} / \Delta x$. Secondorder accuracy is ensured if the numerical derivative and numerical flux derivative satisfy

$$
\begin{gathered}
\frac{u_{j}^{\prime}}{\Delta x}=\left.u_{x}\right|_{x=x_{j}}+O(\Delta x) \\
\frac{f_{j}^{\prime}}{\Delta x}=\left.f(u)_{x}\right|_{u=u\left(x_{j}\right)}+O(\Delta x) .
\end{gathered}
$$

Guidelines are provided in Ref. [7] for determining the numerical derivatives [Eqs. (19) and (20)] to ensure that

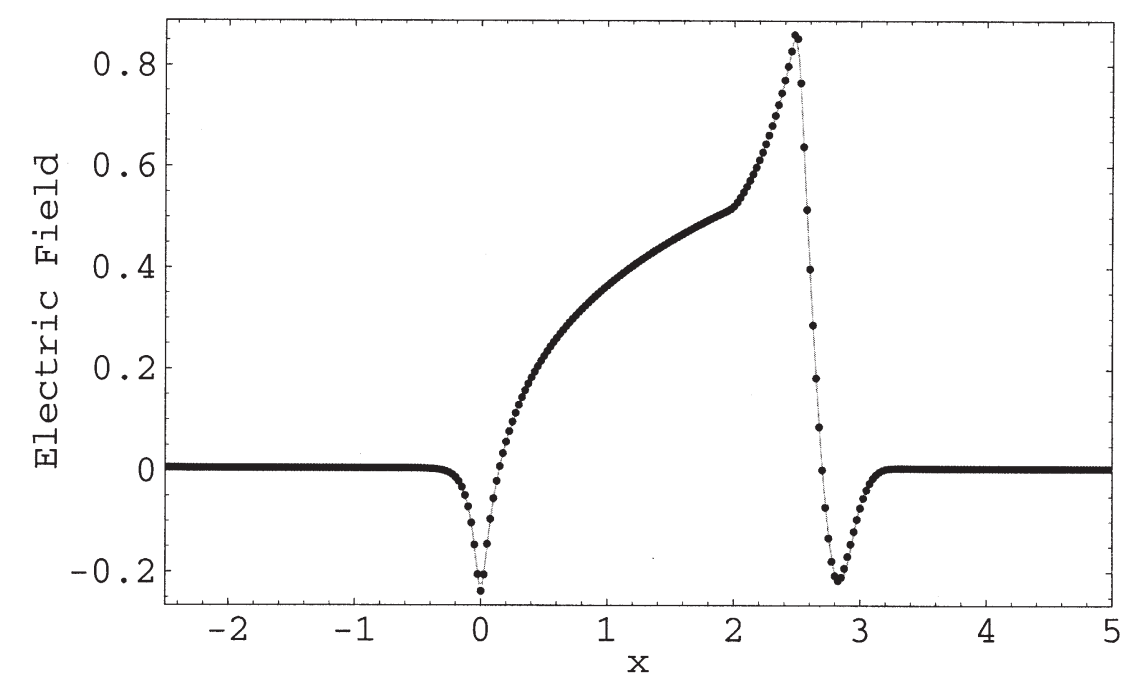

FIGURE 5 Electric field in volts/cm using CLAWPACK (solid) vs. the NTK central scheme (dotted). 
Eq. (15) is TVD (total variation diminishing) in the scalar case. In practice these conditions are met by

$$
\begin{aligned}
& u_{j}^{\prime}=\operatorname{minmod}\left\{\theta \Delta u_{j-\frac{1}{2}}, \frac{1}{2}\left(u_{j+1}-u_{j-1}\right), \theta \Delta u_{j+\frac{1}{2}}\right\} \\
& f_{j}^{\prime}=\operatorname{minmod}\left\{\theta \Delta f_{j-\frac{1}{2}}, \frac{1}{2}\left(f_{j+1}-f_{j-1}\right), \theta \Delta f_{j+\frac{1}{2}}\right\}
\end{aligned}
$$

where $\theta \in[1,2]$ and $\Delta u_{j+1 / 2}=u_{j+1}-u_{j}$. One can instead use the standard flux derivatives $f_{j}^{\prime}=A\left(u_{j}\right) u_{j}^{\prime}$. The NT scheme is easily implemented as a predictorcorrector method

$$
\begin{gathered}
u_{j}\left(t+\frac{\Delta t}{2}\right)=u_{j}(t)-\frac{\lambda}{2} f_{j}^{\prime} \\
u_{j+\frac{1}{2}}(t+\Delta t)=\frac{1}{2}\left(u_{j}(t)+u_{j+1}(t)\right)+\frac{1}{8}\left(u_{j}^{\prime}-u_{j+1}^{\prime}\right) \\
-\lambda\left[f\left(u_{j+1}\left(t+\frac{\Delta t}{2}\right)\right)-f\left(u_{j}\left(t+\frac{\Delta t}{2}\right)\right)\right] .
\end{gathered}
$$

The amount of dissipation produced in the NT and NTK central schemes is inversely proportional to $\theta$.

Because of its simplicity in construction and ability to produce second-order accuracy in smooth regions without requiring explicit knowledge of the characteristic structure of the equations, the NT scheme has recently become a popular choice for simulating semiconductor devices using the hydrodynamic model [8-10]. Here we modify the NT scheme for the hydrodynamic model in two important ways.

First, we adopt the semi-discrete formulation of the NT scheme introduced in Ref. [1], which we will refer to as the NTK scheme. In this formulation, the staggered grid occurs between timesteps. Hence all computation is performed only on the non-staggered gridpoints, eliminating the more complicated staggered grid construction used in all previous applications to semiconductor devices. Another significant advantage is that tighter control volumes are used at shock formations, producing sharper resolution in non-smooth regions. The detailed derivation of this method can be found in Ref. [1] and is briefly summarized below. We note that although different orders of the Runge-Kutta method were applied there to the semi-discrete NTK method, we improve on both the efficiency and complexity by implementing the secondorder Adams-Bashforth scheme. No reduction of accuracy is visible.

Second, in all previous applications of the NT method to the hydrodynamic model, a splitting technique was used to perform temporal integration. The source term step was computed first, and then the advection step was computed using the results from the source term step as initial conditions. This is also the usual approach taken by the Godunov methods for the hydrodynamic model. For steady-state problems, splitting causes the solution to vary slightly because of the additional partial timestep required to complete the method (highly accurate steady-state solutions can be obtained by modifying the method to avoid splitting as steady state is approached-see, e.g. Ref. [11]). Furthermore, two partial steps are required at each time level, increasing the work and computational time. By taking advantage of the Riemann solver free approach in the NT and NTK methods, we are able to avoid splitting.

We will briefly review the NTK semi-discrete formulation. Again we evolve in time the piecewise linear solution [Eq. (14)] at time level $n$ based on cell averages $u_{j}^{n}$ and the reconstructed approximate derivatives $\left(u_{x}\right)_{j}^{n}$. We define

$$
\begin{gathered}
u_{j+\frac{1}{2}}^{+}=u_{j+1}-\frac{\Delta x}{2}\left(u_{x}\right)_{j+1} \\
u_{j+\frac{1}{2}}^{-}=u_{j}+\frac{\Delta x}{2}\left(u_{x}\right)_{j}
\end{gathered}
$$

as the corresponding left and right intermediate cell values. If $\mathscr{C}$ defines a curve in phase space connecting $u_{j+1 / 2}^{-}$and $u_{j+1 / 2}^{+}$via the Riemann fan, then the maximum local speed of propagation at the cell boundary $x_{j+1 / 2}$ is given by

$$
\alpha_{j+\frac{1}{2}}=\max _{u \in \mathscr{C}} \rho\left(\frac{\partial f}{\partial u}\right)
$$

Knowledge of the maximum local speed allows us to obtain tighter control volumes at shock formations. The semi-discrete NTK central scheme for the conservation law [Eq. (11)] can be written in conservation form as

$$
\frac{\mathrm{d}}{\mathrm{d} t} u_{j}(t)=-\frac{H_{j+\frac{1}{2}}-H_{j-\frac{1}{2}}}{\Delta x}
$$

with numerical flux

$$
\begin{aligned}
H_{j+\frac{1}{2}}= & \frac{f\left(u_{j+\frac{1}{2}}^{+}(t)\right)+f\left(u_{j+\frac{1}{2}}^{-}(t)\right)}{2} \\
& -\frac{a_{j+\frac{1}{2}}(t)}{2}\left(u_{j+\frac{1}{2}}^{+}(t)-u_{j+\frac{1}{2}}^{-}(t)\right) .
\end{aligned}
$$

The maximum local speed is typically taken to be

$$
a_{j+\frac{1}{2}}=\max \left\{\rho\left(\frac{\partial f}{\partial u}\left(u_{j}(t)\right)\right), \rho\left(\frac{\partial f}{\partial u}\left(u_{j+1}(t)\right)\right)\right\} \text {. }
$$

As in the case of the original NT scheme, the NTK scheme is TVD in the scalar case if the approximate derivatives $\left(u_{x}\right)_{j}(t)=u_{j}^{\prime} / \Delta x$ satisfy Eq. (21). Additionally, the intermediate flux value $f\left(u_{j+1 / 2}^{+}(t)\right)$ can either be computed as the traditional flux value of $u_{j+1 / 2}^{+}(t)$ or can be approximated by $f_{j+1 / 2}^{+}=f_{j+1}-\Delta x / 2\left(f_{x}\right)_{j+1}$, with $\left(f_{x}\right)_{j+1}$ computed as in Eq. (22). 
We write the hydrodynamic model conservation laws as

$$
q_{t}+f(q)_{x}=G q
$$

where

$$
G=-\left(\begin{array}{ccc}
0 & 0 & 0 \\
\frac{E}{m} & \frac{1}{\tau_{p}} & 0 \\
\frac{3}{2} \frac{T_{0}}{m \tau_{w}} & \frac{E}{m} & \frac{1}{\tau_{w}}
\end{array}\right) .
$$

As noted above, one of the main restrictions in using CLAWPACK is that the hydrodynamic model conservation laws are solved by splitting the system into advection and source term steps. This is necessary because the Riemann problems that must be solved at each timestep for the homogeneous part of the system cannot be coupled with the source terms. Until now, the NT method was also incorporated with a source term step. However, this splitting is completely unnecessary since the NT and NTK schemes do not require Riemann solvers. In fact, the relatively simple structure of the source terms in the hydrodynamic model enables us to use a mixed explicitimplicit method, which both improves the stability restriction on the timestep $\Delta t$ and decreases the total number of timesteps. Furthermore, we rid our steadystate solution of the undesirable side effects of splitting. Our method works as follows:

1. The semi-discrete formulation can be written as

$$
q_{t}=C[q]+G q
$$

where $C[q]$ denotes the spatial discretization of $-f(q)_{x}$ and is computed at the $n$th time level using Eq. (28) as

$$
C\left[q_{j}^{n}\right]=-\frac{H_{j+\frac{1}{2}}^{n}-H_{j-\frac{1}{2}}^{n}}{\Delta x}
$$

2. Poisson's equation (4) is solved using a standard tridiagonal matrix solver to obtain $V_{j}^{n}$ at the $n$th time level for each $j=1, \ldots, N-1$. The electric field is computed as the centered difference approximation $E_{j}^{n}=\left(V_{j+1}^{n}-V_{j-1}^{n}\right) /(2 \Delta x)$ and used in Eq. (32).

3. Time integration is performed by applying the second-order Adams-Bashforth method for the conservation part and the trapezoidal rule for the source terms as

$$
\begin{aligned}
q_{j}^{n+1}= & q_{j}^{n}+\frac{1}{2}\left(3 C\left[q_{j}^{n}\right]-C\left[q_{j}^{n-1}\right]\right)+\frac{1}{2}\left(G q_{j}^{n+1}\right. \\
& \left.+G q_{j}^{n}\right) .
\end{aligned}
$$

Figures 1-5 show the profiles of the steady-state solution. We chose the limiting parameter in Eqs. (21) and (22) to be $\theta=1.2$.

We note that the choice for the limiting value $\theta$ is not arbitrary. Figure 6 illustrates the effects of different values of $\theta$ on the velocity profile of the electron shock wave. The simulations of two-dimensional Riemann problems in Ref. [12] using the NTK central scheme indicate that the best range of $\theta$ for two-dimensional gas dynamics is $1 \leq \theta \leq$ 1.3. The optimal value for resolving the electron shock wave is $\theta=1.2$. For $\theta \geq 1.25$ there is an overshoot in the shock wave, while for $\theta<1.2$ the discontinuity is less sharp.

\section{CONCLUSION}

CLAWPACK resolves the electron shock wave over approximately one or two $\Delta x$ (see Fig. 2), while the NTK central scheme requires three or four $\Delta x$, which is typical of shock resolution for the central schemes. It should be noted however that the shock simulation with the NTK

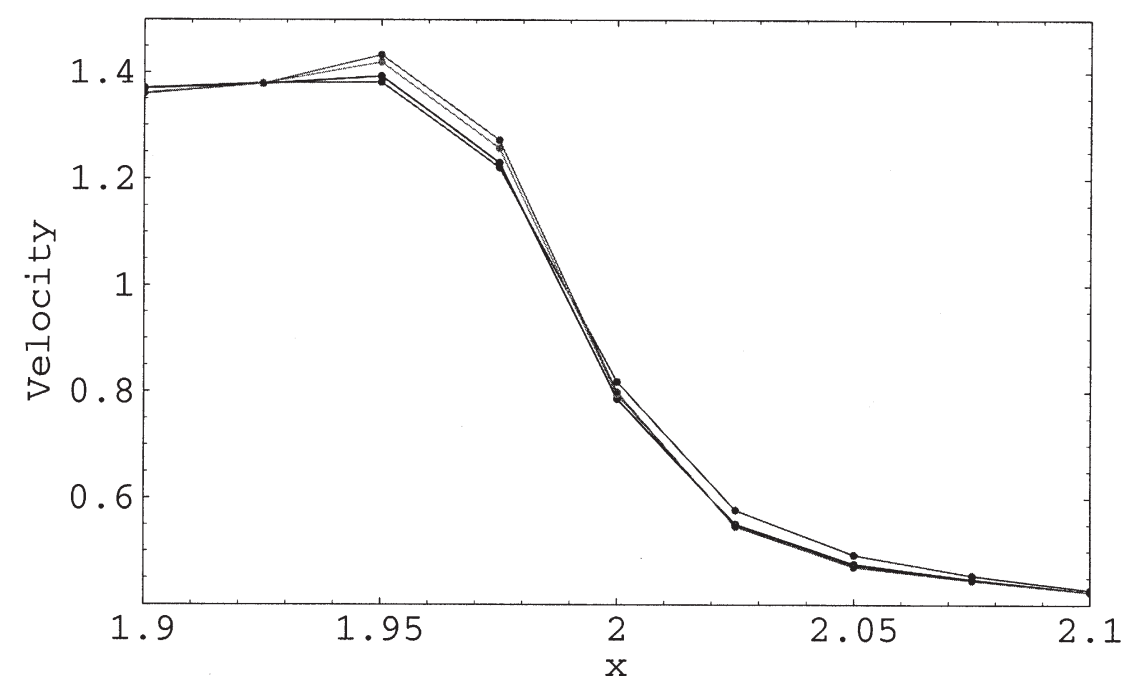

FIGURE 6 Closeup of the shock wave in velocity using the NTK scheme for (from bottom to top at $x=1.95$ ) $\theta=1,1.2,1.25$, and 1.3 . 
scheme exhibits no spurious oscillations or overshoot with $\theta=1.2$.

On the one hand, the central schemes are easier to program than the (higher-order) Godunov methods, are typically faster by a factor of two or more, and can handle source terms without splitting. Further, even minor modifications of the physics of a model can lead to major changes in the eigenstructure of the partial differential equation (PDE) system and in the implementation of higher-order Godunov methods and their Riemann solvers, while the central schemes remain unchanged and are in this sense "universal". On the other hand, it is easy to incorporate source terms in CLAWPACK, and once a system of PDEs is working in CLAWPACK in one spatial dimension, it is transparent to extend the simulations to two and three dimensions, and to make use of automatic mesh refinement.

The current vs. voltage curves-which are the main focus of semiconductor device simulation in the microelectronics industry-predicted by CLAWPACK and the NTK scheme agree to better than six parts in ten thousand, so unless for mathematical reasons one needs to resolve shock waves over 1-2 $\Delta x$, either method may be used to advantage.

\section{Acknowledgements}

We would like to thank Eitan Tadmor for valuable discussions.

\section{References}

[1] Kurganov, A. and Tadmor, E. (2000) "New high-resolution central schemes for nonlinear conservation laws and convection-diffusion equations", Journal of Computational Physics 160, 214-282.

[2] Gardner, C.L. (1991) "Numerical simulation of a steady-state electron shock wave in a submicrometer semiconductor device", IEEE Transactions on Electron Devices 38, 392-398.

[3] Gardner, C.L. (1993) "Hydrodynamic and Monte Carlo simulation of an electron shock wave in a one micrometer $n^{+}-n-n^{+}$diode", IEEE Transactions on Electron Devices 40, 455-457.

[4] Fischetti, M.V. and Laux, S.E. (1988) "Monte Carlo analysis of electron transport in small semiconductor devices including bandstructure and space-charge effects", Physical Review B 38, 9721-9745.

[5] Fatemi, E., Gardner, C.L., Jerome, J.W., Osher, S. and Rose, D.J. (1991) "Simulation of a steady-state electron shock wave in a submicron semiconductor device using high-order upwind methods", Computational Electronics: Semiconductor Transport and Device Simulation (Kluwer Academic Publishers, Boston), pp 27-32.

[6] LeVeque, R.J. (1992) Numerical Methods for Conservation Laws (Birkhauser Verlag, Basel).

[7] Nessyahu, H. and Tadmor, E. (1990) "Non-oscillatory central differencing for hyperbolic conservation laws", Journal of Computational Physics 87, 408-463.

[8] Anile, A.M., Nikiforakis, N. and Pidatella, R.M. (2000) "Assessment of a high resolution centered scheme for the solution of hydrodynamical semiconductor equations", SIAM Journal on Scientific Computing 22, 1533-1548.

[9] Anile, A.M., Romano, V. and Russo, G. (2000) "Extended hydrodynamical model of carrier transport in semiconductors", SIAM Journal on Applied Mathematics 61, 74-101.

[10] Romano, V. and Russo, G. (2000) "Numerical solution for hydrodynamical models of semiconductors", Mathematical Models and Methods in Applied Sciences 10, 1099-1120.

[11] LeVeque, R.J. and Pelanti, M. (2001) "A class of approximate Riemann solvers and their relation to relaxation schemes", Journal of Computational Physics 172, 572-591.

[12] Kurganov, A. and Tadmor, E. "Solution of two-dimensional Riemann problems for gas dynamics without Riemann problem solvers." To appear.

Carl L. Gardner is Professor of Mathematics at Arizona State University. His current research interests lie in classical and quantum semiconductor device simulation, computational fluid dynamics, astrophysical flows, and the modelling and simulation of ion transport in the channels of cellular membranes.

Anne Gelb is Associate Professor of Mathematics at Arizona State University. Her main interests are the study of higher order methods for solving partial differential equations, in particular the applications of spectral methods to discontinuous functions. A large part of her research has been devoted to restoring the exponential convergence qualities to spectral methods for discontinuous functions. Recently she has become interested in central difference schemes applied to conservation laws, with applications to semiconductor device simulation and astrophysical jets.

Justin Hernandez was a graduate student in Mathematics at Arizona State University when this work was completed. Currently he is working as a researcher in computational mathematics. 

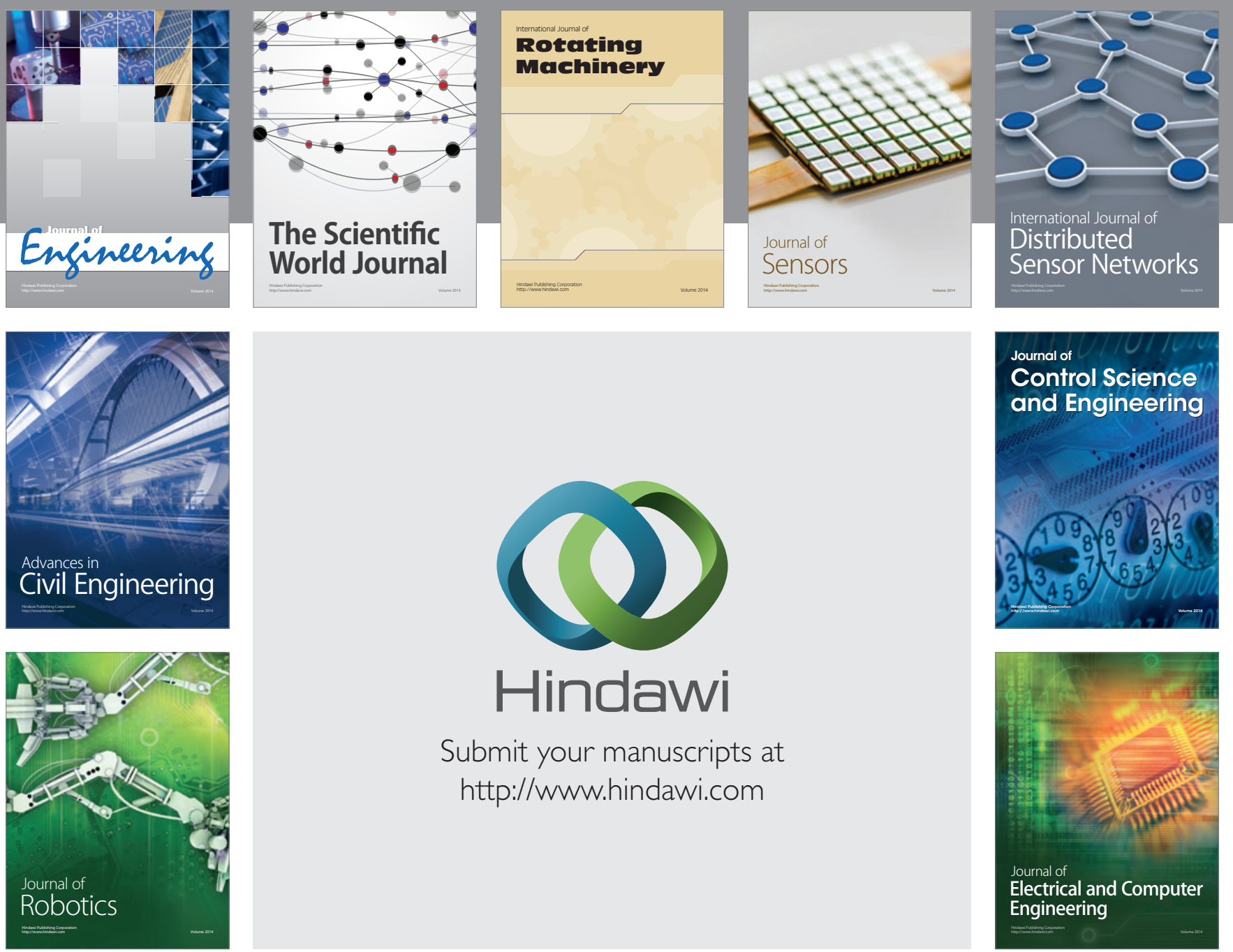

Submit your manuscripts at

http://www.hindawi.com
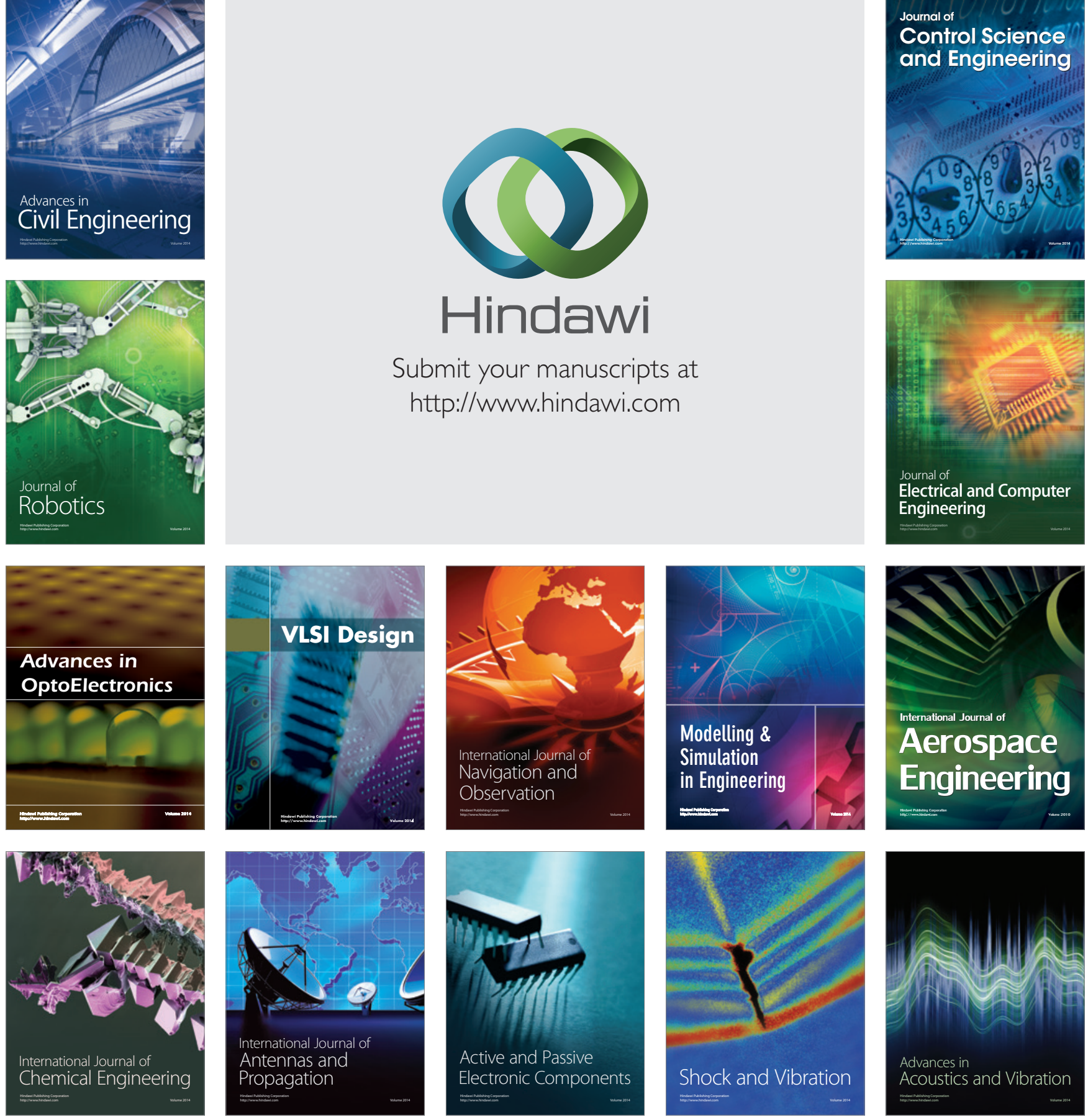